\title{
Impact of Distinctive Jamming Signals on WiMAX System
}

\author{
Priya Mudhol \\ M.Tech student \\ Department of Electronics and Communication \\ Dayananda Sagar college of Engineering \\ Bengaluru
}

\author{
Deepa N. P. \\ Assistant Professor \\ Department of Electronics and Communication \\ Dayananda Sagar college of Engineering \\ Bengaluru
}

\begin{abstract}
In this recent era wireless communication industries have focused on the uses of broadband system having high quality functions. Because of this issue new advances with high transmission capability technologies are designed. The broadband wireless access turns into the best approach to satisfy the high business interest for expanding the web connection. WiMAX ("Worldwide Interoperability for Microwave Access") is a broadband wireless technology is developed to meet the growing demand of increased data rate and accessing the internet at high speeds as well as low cost maintenance. It is based on the IEEE 802.16-2004 standard family. In this system allows using an efficient bandwidth in a wide frequency range, and last mile solution for broadband internet access. The jamming and scrambling are two important threats in WiMAX system. This paper presents the simulation of WiMAX system with and without impact of jamming signals. The performance parameter BER of the system is analyzed in all conditions and the result implies the enormously changes with the utilization of distinctive jamming signals. The aim of this paper is to analyze and simulate WIMAX OFDM system in the presence of interference signals. MATLAB Simulink software is used to simulate and analyze the WIMAX system. The simulation results include the performance analysis based on bit error rate (BER) versus signal to noise rate (Eb/No).
\end{abstract}

\section{Keywords}

WIMAX, Jamming Signals, OFDM, BER, SNR

\section{INTRODUCTION}

WiMAX (Worldwide Interoperability for Microwave Access) is a wireless broadband access in wireless Metropolitan area network. WiMAX belongs to IEEE 802.16 standard family [1]. There are two types of WiMAX, Fixed WiMAX (IEEE 802.16d) and Mobile WiMAX (IEEE 802.16e). WiMAX supports two types of services are Line of Sight which uses frequency range of $10-66 \mathrm{GHz}$ and Non-Line of Sight of 2$11 \mathrm{GHz}$ [2]. It works in point to point (P2P) and point to multi point (P2MP) modes. IEEE 802.16 standard provides Wide range coverage and high speed communication for rural areas, confidence, sophisticate and last mile Broadband facilities. The WiMAX replaces all other wireless broadband technologies and provides excellent solution for Last Mile connection where it is difficult to get by other technologies such as the arrangement of Digital Subscriber Line (DSL) or Cable line unavailable in rural regions. Approximately only 3 miles [5] of distance can cover by utilizing DSL. The coverage area of the Wi-Fi connection is larger than that of DSL but it also has some limitations. WiMAX offers wireless backhaul network it provides internet access to home, small and medium business customer, campus and also to WiFi hot spots and base station. WiMAX provides data rates up to 70Mbps and large geographical area between base station (BS) and subscriber station (SS) covers up to $50 \mathrm{~km}$ for fixed WiMAX and for Mobile WiMaX up to 5-10km [7]. The WiMAX system comprises of two main parts base station or base tower and WiMAX receiver or receiver tower as well as customer premises equipment (CPE).

Physical layer and MAC layer (Medium access control) are the two layers of WiMAX system. The physical layer of WiMAX is based on OFDM. The OFDM modulation technique used is used in WiMAX system because of its spectrally efficient modulation due to its orthogonality principle. The main advantage of using OFDM are bandwidth efficiency, reduces inter-carrier interference (ICI) and also reduces inter symbol interference (ISI).Several researchers thinks that WiMAX can bring the new dimension broadband network for wireless data transmission but there are some limiting factors for transmitting high data rates over medium including fading channel interference. The purpose of this paper is to implement the WiMAX Physical layer based on OFDM and transmit data at lower bit error rate and higher efficient data over channel with different interference signals known as jamming signals. In this paper initially concentrated to analyze the BER parameter performance for WiMAX system and system with jamming signals. The results of each simulated model are compared. The principal concept Noise jamming, Partial-Band noise jamming and tone jamming are inserting to the basically implemented WiMAX transmitter and receiver. Performance results are plotted curves of BER versus SNR. The performance analysis of WiMAX physical layer is done by using MATLAB Simulink software

\section{BASICS OF WiMAX TECHNOLOGY}

In 1998 the Institute of Electrical and Electronic Engineers (IEEE) group was introduced the WiMAX technology based on IEEE802.16 standard to develop an air-interface standard for wireless broadband. Initially this group was focused on the development of LOS based point to multipoint broadband system to operate in the frequency range of $10-66 \mathrm{GHz}$ millimeter wave band. The main goal was to develop WiMAX system to cover the rural areas where DSL and cable infrastructure in not available. In December 2001 the first version of the IEEE 802.16 standard was proposed and approved. The present version of standard IEEE 802.16d Fixed WiMAX was approved in June 2004with transmission scheme of single carrier and FFT size of OFDM 256 and 2048. The IEEE 802.16e Mobile WiMAX was approved in December 2005 and modified as scalable OFDMA with transmission scheme of single carrier, FFT size of OFDM 256 
and scalable OFDMA of FFT size 128,512, 1024 or 2048. These variable sizes of FFT allow for optimum operation of system over a wide range of channel bandwidth and radio condition.

\section{WiMAX WITH DISTINCTIVE JAMMING SIGNAL MODEL}

In this paper presents the WiMAX IEEE 802.16-d physical layer based on OFDM simulation model implemented and interference signal are inserted at channel. The connection set up of physical layer is responsible for transmitting the bit sequence between the communication devices. There are two types of transmission techniques in WiMAX 802.16 PHY layer, they are OFDM and OFDMA and these both have frequency band of $2-11 \mathrm{GHz}$ and uses the TDD and FDD. WiMAX based on OFDM provides high speed data transmission and for multimedia applications. The simulation model mainly consists of three stages, Transmitter, Channel and Receiver.

The specification of WiMAX physical layer based OFDM are given as the transmitter block consists of Randomized data, FEC, rectangular QAM-16 modulation and OFDM transmitter (IFFT) and Additive White Gaussian Noise is used as channel and the receiver consist of OFDM receiver (FFT), rectangular QAM-16 demodulator, Decoder (Viterbi decoder, RS decoder). The error rate of bit is calculated between the output of randomized data and the output of Reed-Solomon decoder. The jamming signals are classified as follows: Noise and Tone jamming. According to how incomprehensible a small amount of the communication framework frequency band of the Noise jamming classified into barrage and narrow-band jamming signal. Tone jamming incorporates in to Single tone and Multi-tone jamming which impacts most on the general execution of the system.
The noise jamming method is to embed a disturbance signal into the WiMAX system so that the needed signal is totally submerged by the obstruction. Such kind of jamming is known as denial jamming. Jamming signal $\mathrm{j}(\mathrm{t})$ equation is described as

$$
\mathrm{j}(\mathrm{t})=\mathrm{An}(\mathrm{t}) \sin (\omega \mathrm{ct}+\varphi)
$$

Where $\omega c t$ is carrier frequency, $\varphi$ is initial phase, $A$ is amplitude and $n(t)$ is denotes noise of channel with the mean value of zero.

Partial-Band Noise Jamming (PBNJ) is method of limiting the band of applied frequency. Its prescribed model as zero mean Additive Gaussian Noise (AGN) with most of the concentrated power is at a restricted segment of complete bandwidth. This is considered as more effective than noise jamming as more power is injected in smaller bandwidth to interfere with the original signal. In implementation considering the band restricted Gaussian noise (PBNJ) can be generated by passing the additive white Gaussian noise (AWGN) over a filter of preferred type and bandwidth. Such a filtering operation eliminates the whiteness of the noise and band limits.

Tone jamming is a method of injecting the number of signals tone in the WiMAX system channel to disturb the performance of the entire system. This can be divided into Single tone jamming and Multi tone jamming.

Single tone jamming signal can only interfere at one frequency point. Multiple tones jamming signal are inserted to disturb at parts of frequency points in the WiMAX system.

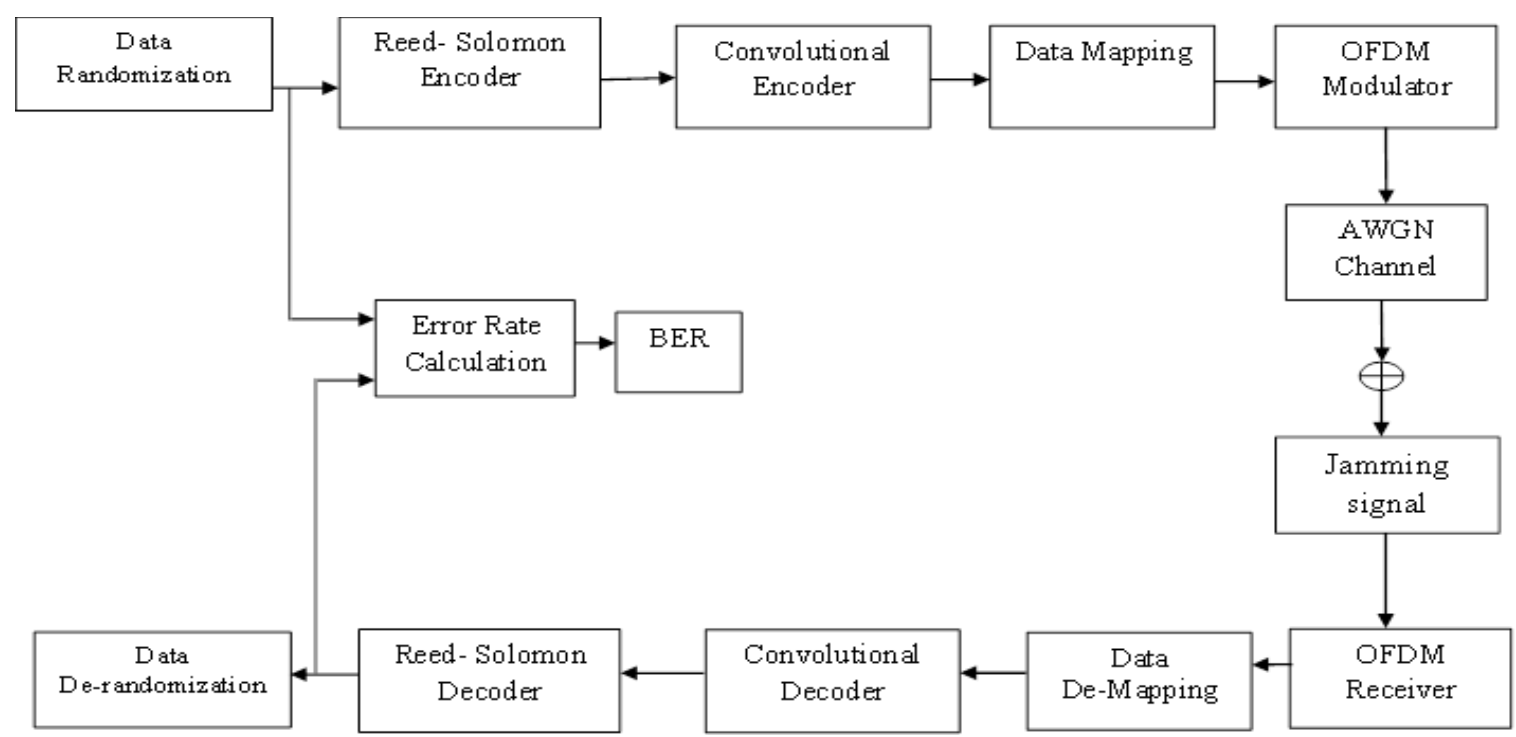

Fig 1: Block diagram of WiMAX physical layer with jamming signal

\section{SIMULATION RESULTS}

The software MATLAB/Simulink is used to build simulation model for WiMAX system. Here calculated the BER parameter to estimate the built system performance. Different jamming signals are introduced into the designed model along with AWGN channel. The techniques of Reed Solomon encoder and decoder and convolutional encoder and decoder Rectangular QAM-16 Modulation, OFDM Modulation and are applied for improving the communication performance for the data transfer system. The performance of WiMAX system under impact of distinctive jamming signal are determined by calculating the bit error rate and signal to noise ratio $(\mathrm{Eb} / \mathrm{No})$ between the un-modulated signal data at the receiving end.

The simulation result consists of WiMAX Physical layer simulation model with the presence of jamming signals and the graphs are plotted for each jamming signal simulation model at BER vs. Eb/No. the calculation is carried out by utilizing below mentioned equations: 
$\mathrm{BER}=$ (received number of bits with an Error) / (transmitted number of information bits)

Signal-to- noise ratio $(\mathrm{Eb} / \mathrm{No})=10 \log 10($ Signal Power /

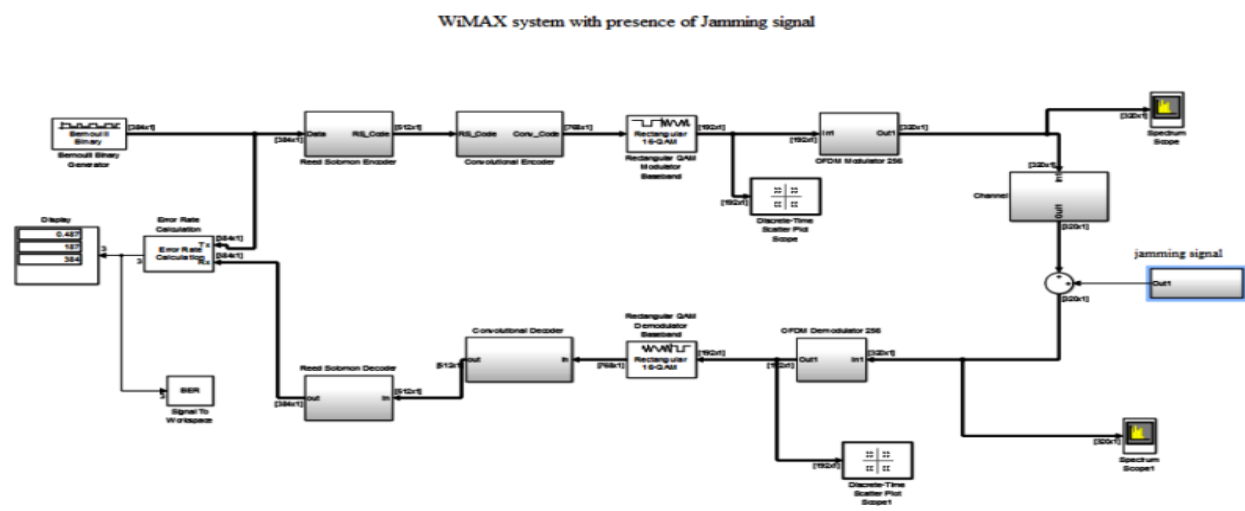

Fig 2: WiMAX Physical Layer with jamming signals Simulink Model in MATLAB

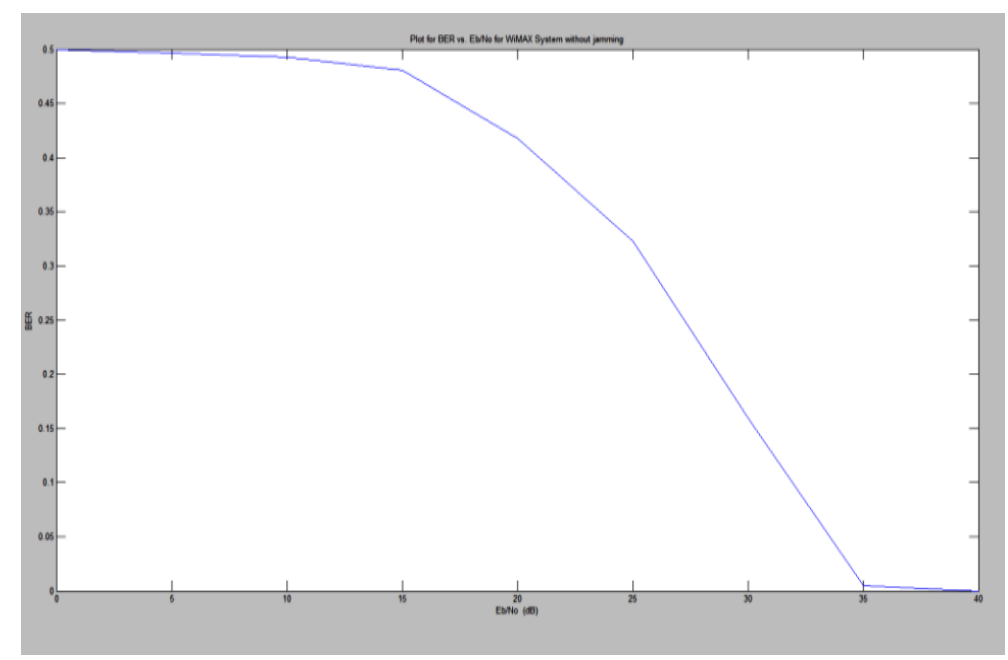

Fig 3: BER plot for WiMAX Physical layer Model

The Fig 3 shows the plot of BER vs. Eb/ No for WiMAX physical layer model without the presence of jamming signals. The results of this graph are compared with the simulated results of jamming signals graphs. Each jamming signal is individually injected to the WiMAX system and calculated the Bit error rate performance at different signal to noise ratio $(\mathrm{Eb} / \mathrm{No})$.

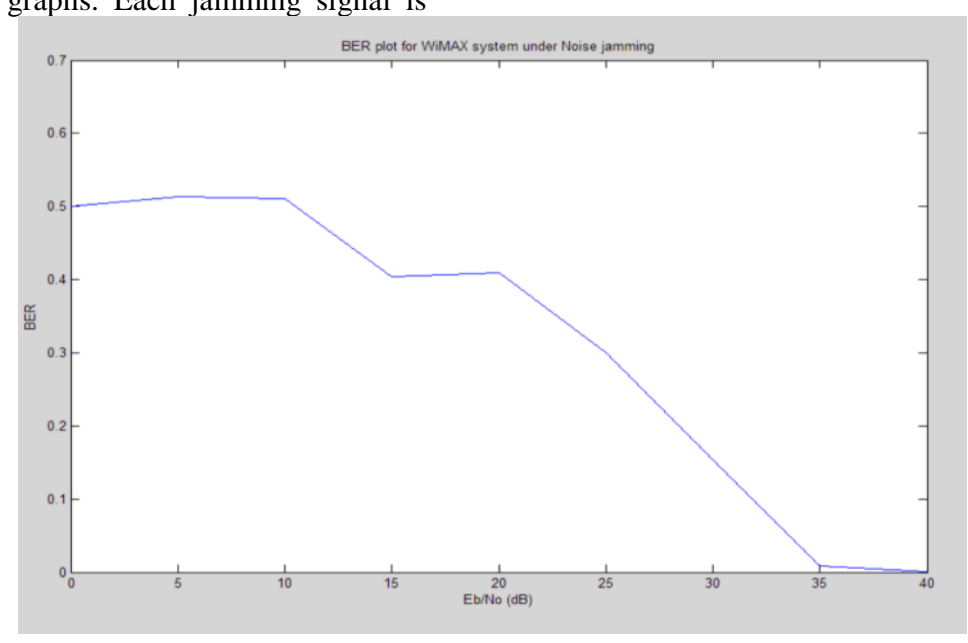

Fig 4: BER plot for WiMAX Physical layer Model at Noise jamming 
The Fig 4 shows the BER vs. Eb/No plot for WiMAX system after inserting the noise jamming signal with the AWGN channel. The noise jamming slightly varies the performance of WiMAX system due the presence of more disturbance signal at the receiver
The Fig 5 shows the BER vs. Eb/No plot for WiMAX system after inserting the Partial-Band Noise jamming signal with the AWGN channel. This jamming signal differs enormously the performance of WiMAX system.

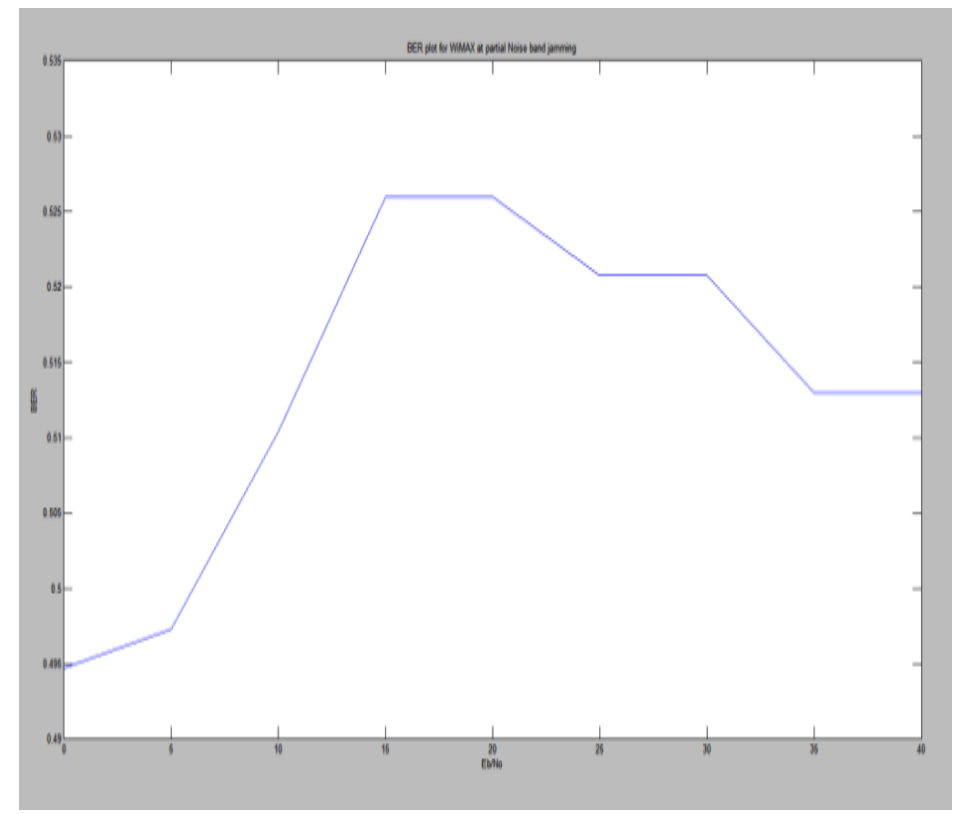

Fig 5: BER plot for WiMAX Physical layer Model at Partial band noise jamming

The tone jamming is inserted in two different ways; first the single carrier frequency tone of $1 \mathrm{MHz}$ signal is inserted at channel of WiMAX system with different simulation time from module. This method of performance is known as single tone jamming. The plot of BER vs. Eb/No plot for WiMAX system with single tone jamming single are plotted at fig 6 .
Another way is inserting different carrier frequencies as $1 \mathrm{MHz}, 5 \mathrm{MHz}, 70 \mathrm{MHz}$ and $100 \mathrm{MHz}$ at different simulation time of module. This method of performance is known Multitone-jamming. The output plot of BER vs. Eb/No at Multi tone jamming signals is plotted at figure 7 .

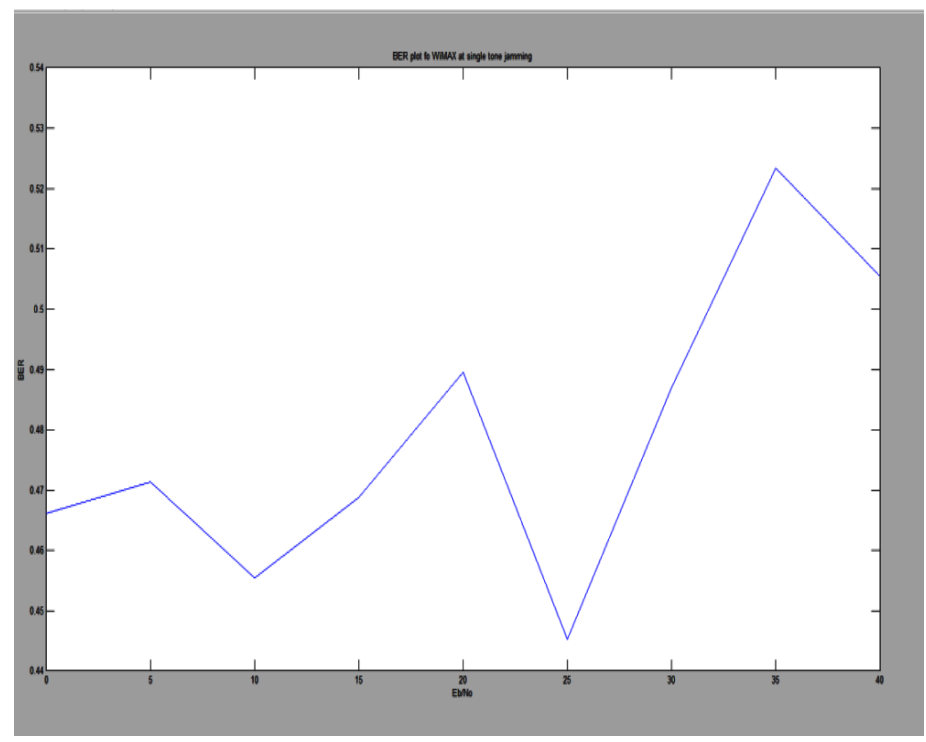

Fig 6: BER plot for WiMAX Physical layer Model at single tone jamming 


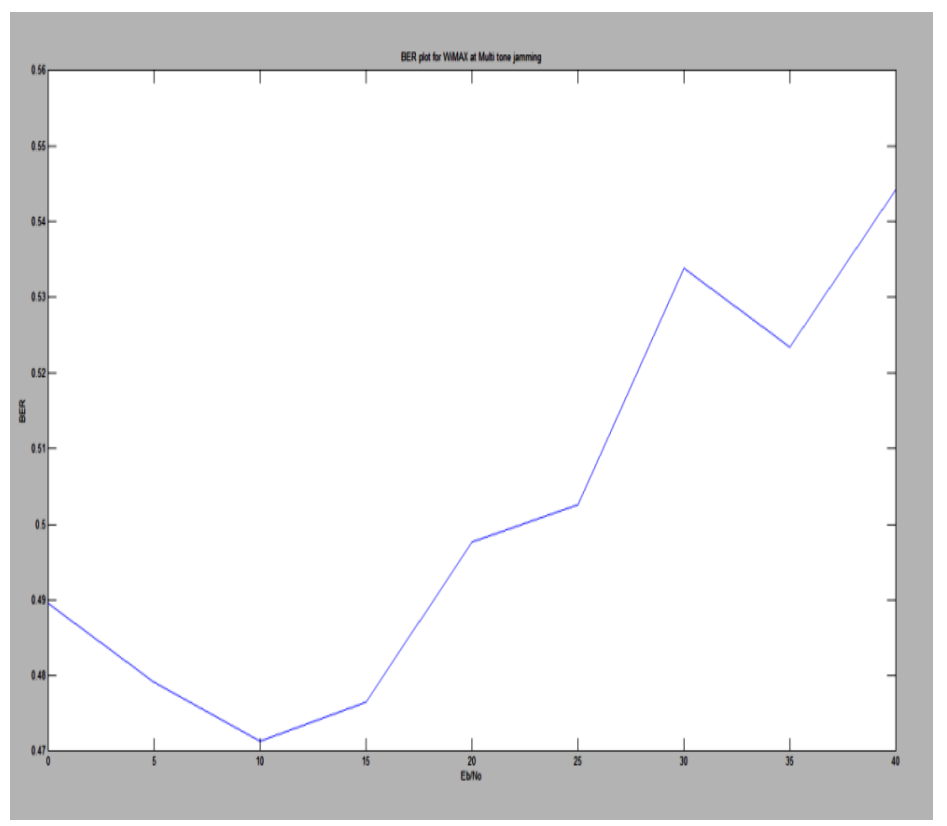

Fig 7: BER plot for WiMAX Physical layer Model at Multi tone jamming

Table 1: Comparison of performance of BER at different signal to noise ratio on WiMAX system utilizing distinctive jamming signals

\begin{tabular}{|c|c|c|c|c|c|}
\hline $\begin{array}{c}\text { Eb/No in } \\
\mathrm{dB}\end{array}$ & $\begin{array}{c}\text { BER for } \\
\text { IVilLAX } \\
\text { șstem }\end{array}$ & $\begin{array}{c}\text { BER for } \\
\text { presence of } \\
\text { Noise } \\
\text { Jamming }\end{array}$ & $\begin{array}{c}\text { BER for } \\
\text { presence of } \\
\text { PBNJ }\end{array}$ & $\begin{array}{c}\text { BER for } \\
\text { presence of } \\
\text { Single tone } \\
\text { Jamming }\end{array}$ & $\begin{array}{c}\text { BER for } \\
\text { presence of } \\
\text { Nulti-tone } \\
\text { Jamming }\end{array}$ \\
\hline 0 & 0.5001 & 0.5 & 0.4947 & 0.4661 & 0.4895 \\
\hline $\mathbf{5}$ & 0.4965 & 0.513 & 0.4973 & 0.4714 & 0.4791 \\
\hline 10 & 0.4929 & 0.5104 & 0.5104 & 0.4554 & 0.4713 \\
\hline 15 & 0.4809 & 0.4036 & 0.5260 & 0.4688 & 0.4765 \\
\hline 20 & 0.4176 & 0.4089 & 0.5260 & 0.4896 & 0.4976 \\
\hline $\mathbf{2 5}$ & 0.3228 & 0.2995 & 0.5208 & 0.453 & 0.5026 \\
\hline
\end{tabular}

This table gives the comparison results of WiMAX system with each jamming signal inserted at different SNR values and Bit error rate of each module is tabulated.

\section{CONCLUSION}

The aim of this paper is to build WiMAX communication system model, with the help of MATLAB to carry out simulation for predicting its system receiving characteristics; to explore techniques of Jamming effects on the wireless communication performance. This model represents the analysis of the WiMAX Performance by using AWGN channel with distinctive jamming signal and is evaluated over OFDM system. The simulated WiMAX physical layer performance parameter BER mainly depends on the channel conditions and the injected jamming signal. The impact of Multi-tone and Partial-band noise jamming are higher compared to the single tone and noise jamming on the WiMAX system. Noise jamming gives the worst result for channel condition. Single tone jamming is not more effective as Multi-tone jamming on WiMAX system
Future scope attempts can be made to overcome the interference signal on utilizing the different channel conditions. Channel model must be built to eliminate interference or tolerate jamming signals. This phenomenon may increases the better BER performance in WiMAX communication system.

\section{ACKNOWLEDGMENTS}

I am glad to express my sincere thanks to Assistant professor Ms. DEEPA.N.P and Mr. SIVAKUMAR .S Senior Research Member at Bharat Electronic Limited, Bengaluru; who offered me valuable and constructive tips for my work.

\section{REFERENCES}

[1] Mikka Juhani Husso, "Performance Analysis of WiMAX system under jamming". Helsinki University of technology, December 2006.

[2] Jaun Li, Sven-Gustav Haggman,"Performance of IEEE 802.16 Based system in Jamming Environment and its Improvement with Link Adoption". The 17th Annual IEEE International symposium on personal, Indoor and Mobile Radio Communication (IEEE 2006).

[3] Mohammad Azizul Hasan, Master thesis, "Performance Evaluation of WiMAX/IEEE 802.16d OFDM Physical Layer", June 2007.

[4] A.Roca, "Implementation of WiMAX simulator in Simulink" (2007), Engineering Institute-Vienna, February 2007.

[5] Ali Hasan, Ali Al Qahtani, "Analysis and simulation of MC-CDMA for WiMAX system technology/IEEE standard 802.16” King Abdulaziz university, June 2009.

[6] Rakesh kumar Jha and Dr.Upena Dalal "Performance analysis under the influence of jamming for WiMAX systems", Published in IEEE- 2011.

[7] Payaswin $\mathrm{p}$ and Manjaiah D.H "Analysis of Effect of cyclic prefix on Data rates in OFDM Modulation techniques" from Mangalore University October 2012.

[8] Vivek kumar Jaiswal, Mr.G.S. Tirupathi "Performance Evaluation of WiMAX based OFDMA System in 
Multipath Fading Channel" published at IJAREEIE in June 2013

[9] HAO XIE from Chalmers university "Modeling of Highspeed data link for Wireless communication on Ship platform" 2013.

[10] Muhammad Aamer, Abdual Maalik "Performance analysis of WiMAX Physical layer under scrambling security threat using MATLAB simulation", published at International Journal of computer science and telecommunication in 2013.

[11] Sweety Sangwan Gulia, Mr. Ankit "Performance Analysis of WIMAX Based OFDM System Using
Various Modulation Techniques" International Journal of Engineering and Innovative Technology (IJEIT) Volume 3, Issue 1, July 2013

[12] Md. Anamul Islam, B. Md. Abdul Kader, C. Md. Julkarnain "BER Performance Analysis of a Real Data Communication through WiMAX-PHY Layer over an AWGN and Fading Channels" International Journal of Electrical \& Computer Sciences IJECS-IJENS Vol: 10 No: 04

[13] WiMAX Forum, http://www.wimaxforum.org-2015 